\title{
The $p$ th moment exponential stability of stochastic cellular neural networks with impulses
}

\author{
Xiaoai Li ${ }^{1^{*}}$, Jiezhong Zou' and Enwen Zhu ${ }^{2^{*}}$
}

"Correspondence:

xiaoaili@csu.edu.cn; engwenzhu@126.com

${ }^{1}$ School of Mathematics and Statistics, Central South University, Changsha, Hunan 410083, China

${ }^{2}$ School of Mathematics and Computational Science, Changsha University of Science and Technology, Changsha, Hunan 410004 , China

\begin{abstract}
This paper studies the $p$ th moment exponential stability of stochastic cellular neural networks with time-varying delays under impulsive perturbations. Based on the Lyapunov function, Razumikhin theory, stochastic analysis and differential inequality technique, criteria on the $p$ th moment exponential stability of this model are derived. These results generalize and improve some of the existing ones. A numerical example illustrates the effectiveness and improvements of our results.
\end{abstract}

Keywords: pth moment exponentially stable; stochastic cellular neural network; impulses; Razumikhin theory

\section{Introduction}

Since Chua and Yang [1, 2] introduced a cellular neural network in 1988, it has received great attention because of its various applications such as classification of patterns, associative memories and optimization, etc. It should be pointed out that time delays are commonly encountered in real systems, which are the source of oscillation and instability both in biological and artificial neural networks, hence it is necessary and important to discuss the delayed cellular neural networks models. Up to now, many results on the stability of delayed neural networks have been developed [3-5]. In fact, in real nervous systems, synaptic transmission is a noisy process brought on by random fluctuations from the release of neurotransmitters and other probabilistic causes. Therefore, noise is unavoidable and should be taken into consideration in modeling. Some recent results of stochastic cellular neural networks with delays can be found in [6-15].

On the other hand, it is noteworthy that the state of electronic networks is often subjected to some phenomenon or other sudden noises. On that account, the electronic networks will experience some abrupt changes at certain instants that in turn affect dynamical behaviors of the systems. Therefore, it is necessary to take both stochastic effects and impulsive perturbations into account on dynamical behaviors of delayed neural networks. In recent years, the dynamic analysis of neural networks with impulsive and stochastic effects has been an attractive topic for many researchers, and a large number of stability criteria of these systems have been reported; see [3, 4, 10-12, 16, 17].

(c) $2013 \mathrm{Li}$ et al.: licensee Springer. This is an Open Access article distributed under the terms of the Creative Commons Attribution License (http://creativecommons.org/licenses/by/2.0), which permits unrestricted use, distribution, and reproduction in any medium, provided the original work is properly cited. 
In [8], Sun et al. investigated the following stochastic cellular neural networks model with time-varying delays:

$$
\begin{aligned}
d x_{i}(t)= & {\left[-c_{i} x_{i}(t)+\sum_{j=1}^{n} a_{i j} f_{j}\left(x_{j}(t)\right)+\sum_{j=1}^{n} b_{i j} g_{j}\left(x_{j}\left(t-\tau_{j}(t)\right)\right)+I_{i}\right] d t } \\
& +\sum_{l=1}^{m} \sigma_{i l}\left(t, x_{i}(t), x_{i}\left(t-\tau_{i}(t)\right)\right) d w_{l}(t),
\end{aligned}
$$

where $x_{i}(t)$ denotes the potential (or voltage) of a cell $i$ at time $t$; $\Lambda=\{1,2, \ldots, n\}, n$ corresponds to the number of units in a neural network; $f_{j}(\cdot), g_{j}(\cdot)$ are activation functions; $c_{i}>0$ denotes the rate at which a cell $i$ resets its potential to the resting state when isolated from other cells and inputs; $a_{i j}$ and $b_{i j}$ denote the strengths of connectivity between cells $i$ and $j$, respectively; $I_{i}$ denotes the external bias on the $i$ th unit, $\tau_{i}(t)$ satisfies $0 \leq \tau_{i}(t) \leq \tau$ and it is a transmission delay. $\sigma(t, x, y)=\left(\sigma_{i l}\left(t, x_{i}, y_{i}\right)\right)_{n \times m} \in \mathbb{R}^{n \times m}$ is the diffusion coefficient matrix and $\sigma_{i}\left(t, x_{i}, y_{i}\right)=\left(\sigma_{i 1}\left(t, x_{i}, y_{i}\right), \ldots, \sigma_{i m}\left(t, x_{i}, y_{i}\right)\right)$ is the $i$ th row vector of $\sigma(t, x, y)$. $w(t)=\left(w_{1}(t), w_{2}(t), \ldots, w_{m}(t)\right)^{T}$ is an $m$-dimensional Brownian motion defined on a complete probability space $(\Omega, \mathcal{F}, P)$ with a natural filtration $\left\{F_{t}\right\}_{t \geq 0}$.

They investigated the $p$ th moment exponential stability with the help of the method of variation parameter and inequality technique, where $p \geq 2$ denotes a positive constant. More precisely, they established the following fundamental assumptions:

(H) For each $j=1,2, \ldots, n, \tau_{j}(t)$ is a differentiable function, namely, there exists $\zeta$ such that

$$
\dot{\tau}_{j}(t) \leq \zeta<1
$$

(H1) Functions $f_{j}(\cdot)$ and $g_{j}(\cdot)$ are Lipschitz-continuous on $\mathbb{R}$ with Lipschitz constants $L_{i}>0, N_{i}>0$. That is, for all $x, y \in \mathbb{R}, i \in \Lambda$,

$$
\left|f_{i}(x)-f_{i}(y)\right| \leq L_{i}|x-y|, \quad\left|g_{i}(x)-g_{i}(y)\right| \leq N_{i}|x-y| .
$$

(H2) There exist nonnegative constants $l_{i}$, $e_{i}$, such that for all $x, y, x^{\prime}, y^{\prime} \in \mathbb{R}, i \in \Lambda$,

$$
\left[\sigma_{i}\left(t, x^{\prime}, y^{\prime}\right)-\sigma_{i}(t, x, y)\right]\left[\sigma_{i}\left(t, x^{\prime}, y^{\prime}\right)-\sigma_{i}(t, x, y)\right]^{T} \leq e_{i}\left|x^{\prime}-x\right|^{2}+l_{i}\left|y^{\prime}-y\right|^{2} .
$$

Huang et al. [6] studied (1.1) and obtained the $p$ th moment exponential stability by using Dini-derivative and Halanay-type inequality without assumption $(\mathrm{H})$. When $k_{1}^{\prime}>k_{2}^{\prime}$, the equilibrium point of the system (1.1) is $p$ th moment exponentially stable, where

$$
\begin{aligned}
k_{1}^{\prime}= & \min _{1 \leq i \leq n}\left\{p c_{i}-(p-1) \sum_{j=1}^{n}\left(L_{j}\left|a_{i j}\right|+N_{j}\left|b_{i j}\right|\right)-\sum_{j=1}^{n} \frac{\mu_{j}}{\mu_{i}}\left|a_{j i}\right| L_{i}-\sum_{j=1}^{n} \frac{(p-2)(p-1)}{2} e_{j}\right. \\
& \left.-\sum_{j=1}^{n} \frac{\mu_{j}}{\mu_{i}} e_{i}-\sum_{j=1}^{n} \frac{(p-1)(p-2)}{2} l_{j}\right\}, \\
k_{2}^{\prime} & =\max _{1 \leq i \leq n}\left\{N_{i} \sum_{j=1}^{n} \frac{\mu_{j}}{\mu_{i}}\left|b_{j i}\right|+\frac{\mu_{j}}{\mu_{i}}(p-1) l_{i}\right\},
\end{aligned}
$$

where $\mu_{i}(i \in \Lambda)$ are positive constants. 
Very recently, Li [11] generalized (1.1); he considered a stochastic cellular neural network under impulsive perturbations. The condition $k_{1}^{\prime}>k_{2}^{\prime}$ is also needed to ensure exponential stability in mean square.

We have a question whether the condition $k_{1}^{\prime}>k_{2}^{\prime}$ in the theorems $[6,11,12]$ is an essential condition or not for the equilibrium point of (1.1) to be $p$ th moment exponentially stable.

In this paper, we solve this question and obtain the improved version of the $p$ th moment exponential stability by applying Lyapunov functions, Razumikhin theory and inequality technique. An example is also provided to illustrate the effectiveness of the new results.

\section{Preliminaries}

In this paper, we study stochastic cellular neural networks with impulses described by the delayed differential equations

$$
\left\{\begin{aligned}
d x_{i}(t)= & {\left[-c_{i} x_{i}(t)+\sum_{j=1}^{n} a_{i j} f_{j}\left(x_{j}(t)\right)+\sum_{j=1}^{n} b_{i j} g_{j}\left(x_{j}\left(t-\tau_{j}(t)\right)\right)+I_{i}\right] d t } \\
& +\sum_{l=1}^{m} \sigma_{i l}\left(t, x_{i}(t), x_{i}\left(t-\tau_{i}(t)\right)\right) d w_{l}(t), \quad t \neq t_{k}, \\
\Delta x_{i}\left(t_{k}\right)= & p_{i k}\left(x\left(t_{k}\right)\right)=x_{i}\left(t_{k}\right)-x_{i}\left(t_{k}^{-}\right), \quad k \in \mathbb{Z}^{+}, i \in \Lambda,
\end{aligned}\right.
$$

where $\left\{t_{k}\right\}$ is the time sequence and satisfies $0=t_{0}<t_{1}<t_{2}<\cdots<t_{k}<t_{k+1} \cdots, \lim _{k \rightarrow \infty} t_{k}=$ $\infty ; x_{t}(s)=x(t+s), s \in[-\tau, 0]$. For $k=1,2, \ldots, p_{i k}\left(x\left(t_{k}\right)\right)$ represents the abrupt change of the state $x_{i}(t)$ at the impulsive moments $t_{k}$.

System (2.1) is supplemented with the initial condition given by

$$
x_{t_{0}}(s)=\psi(s), \quad s \in[-\tau, 0]
$$

where $\psi(s)$ is $\mathcal{F}_{0}$-measurable and continuous everywhere except at a finite number of points $t_{k}$, at which $\psi\left(t_{k}^{+}\right)$and $\psi\left(t_{k}^{-}\right)$exist and $\psi\left(t_{k}^{+}\right)=\psi\left(t_{k}\right)$.

Let $P C^{1,2}\left(\left[t_{k}, t_{k+1}\right) \times \mathbb{R}^{n} ; \mathbb{R}^{+}\right)$denote the family of all nonnegative functions $V(t, x)$ on $\left[t_{k}, t_{k+1}\right) \times \mathbb{R}^{n}$ which are continuous once differentiable in $t$ and twice differentiable in $x$. If $V(t, x) \in P C^{1,2}\left(\left[t_{k}, t_{k+1}\right) \times \mathbb{R}^{n} ; \mathbb{R}^{+}\right)$, define an operator $\mathcal{L} V$ associated with (2.1) as

$$
\begin{aligned}
\mathcal{L} V(t, x)= & V_{t}(t, x)+\sum_{i=1}^{n} V_{x_{i}}(t, x)\left[-c_{i} x_{i}(t)+\sum_{j=1}^{n} a_{i j} f_{j}\left(x_{j}(t)\right)+\sum_{j=1}^{n} b_{i j} g_{j}\left(x_{j}\left(t-\tau_{j}(t)\right)\right)+I_{i}\right] \\
& +\frac{1}{2} \operatorname{trace}\left[\sigma^{T} V_{x x}(t, x) \sigma\right],
\end{aligned}
$$

where

$$
V_{t}(t, x)=\frac{\partial V(t, x)}{\partial t}, \quad V_{x_{i}}(t, x)=\frac{\partial V(t, x)}{\partial x_{i}}, \quad V_{x x}(t, x)=\left(\frac{\partial V(t, x)}{\partial x_{i} \partial x_{j}}\right)_{n \times n} .
$$

Throughout this paper, the following standard hypothesis is needed:

(H3) $p_{i}\left(x_{i}\left(t_{k}\right)\right)=-\beta_{i k}\left(x_{i}\left(t_{k}\right)-x_{i}^{*}\right)$, where $x_{i}^{*}$ is the equilibrium point of (2.1) with the initial condition (2.2), $\beta_{i k}$ satisfies $\left|1-\beta_{i k}\right| \leq d_{k}, d_{k}$ is a positive constant. 
Let $y_{i}(t)=x_{i}(t)-x^{*}$, then $(2.1)$ can be written by

$$
\left\{\begin{aligned}
d y_{i}(t)= & {\left[-c_{i} y_{i}(t)+\sum_{j=1}^{n} a_{i j} \tilde{f}_{j}\left(y_{j}(t)\right)+\sum_{j=1}^{n} b_{i j} \tilde{g}_{j}\left(y_{j}\left(t-\tau_{j}(t)\right)\right)\right] d t } \\
& +\sum_{l=1}^{m} \tilde{\sigma}_{i l}\left(t, y_{i}(t), y_{i}\left(t-\tau_{i}(t)\right)\right) d w_{l}(t), \quad t \neq t_{k}, \\
\Delta y_{i}\left(t_{k}\right)= & \tilde{p}_{i k}\left(y\left(t_{k}\right)\right), \quad k \in Z^{+}, i \in \Lambda,
\end{aligned}\right.
$$

where

$$
\begin{aligned}
& \tilde{f}_{j}\left(y_{j}(t)\right)=f_{j}\left(y_{j}(t)+x_{j}^{*}\right)-f_{j}\left(x_{j}^{*}\right), \quad \tilde{g}_{j}\left(y_{j}\left(t-\tau_{j}(t)\right)\right)=g_{j}\left(y_{j}\left(t-\tau_{j}(t)\right)+x_{j}^{*}\right)-g_{j}\left(x_{j}^{*}\right), \\
& \tilde{\sigma}_{i j}\left(t, y_{i}(t), y_{i}\left(t-\tau_{i}(t)\right)\right)=\sigma_{i j}\left(t, y_{i}(t)+x_{i}^{*}, y_{i}\left(t-\tau_{i}(t)\right)+x_{i}^{*}\right)-\sigma_{i j}\left(t, x_{i}^{*}, x_{i}^{*}\right), \\
& \tilde{p}_{i k}\left(y\left(t_{k}\right)\right)=p_{i k}\left(y\left(t_{k}\right)+x^{*}\right)-p_{i k}\left(x^{*}\right) .
\end{aligned}
$$

In the following, for further study, we first give the following definitions and lemmas.

$\|x\|$ denotes a vector norm defined by

$$
\|x\|^{p}=\sum_{i=1}^{n}\left|x_{i}(t)\right|^{p}, \quad\|\psi\|_{\tau}=\sup _{s \in[-\tau, 0]}\|\psi(s)\| .
$$

Definition 2.1 (Mao [18]) The equilibrium point $x^{*}=\left(x_{1}^{*}, x_{2}^{*}, \ldots, x_{n}^{*}\right)^{T}$ of the system (2.1) is said to be $p$ th moment exponentially stable if there exist $\lambda>0$ and $M>0$ such that

$$
E\left\|x(t)-x^{*}\right\|^{p} \leq M\left\|x_{0}-x^{*}\right\|^{p} e^{-\lambda\left(t-t_{0}\right)}, \quad \forall t \geq t_{0}, \forall x_{0} \in \mathbb{R}^{n}, x\left(t_{0}\right)=x_{0} .
$$

In such a case,

$$
\limsup _{t \rightarrow \infty} \frac{1}{t} \ln E\left(\left\|x(t)-x^{*}\right\|^{p}\right) \leq-\lambda .
$$

The right-hand side of (2.5) is commonly known as the $p$ th moment Lyapunov exponent of this solution.

When $p=2$, it is usually said to be exponentially stable in mean square.

Lemma 2.2 If $a_{i}(i=1,2, \ldots, p)$ denote $p$ nonnegative real numbers, then

$$
a_{1} a_{2} \cdots a_{p} \leq \frac{a_{1}^{p}+a_{2}^{p}+\cdots+a_{p}^{p}}{p}
$$

where $p \geq 1$ denotes an integer.

A particular form of (2.6), namely

$$
a_{1}^{p-1} a_{2} \leq \frac{(p-1) a_{1}^{p}}{p}+\frac{a_{2}^{p}}{p}, \quad \text { for } p=1,2,3, \ldots
$$




\section{Main result}

Theorem 3.1 Assume that (H1)-(H3) hold; furthermore, let

$$
\begin{aligned}
k_{1}= & \min _{1 \leq i \leq n}\left\{p c_{i}-(p-1) \sum_{j=1}^{n}\left(L_{j}\left|a_{i j}\right|+N_{j}\left|b_{i j}\right|\right)\right. \\
& \left.-\sum_{j=1}^{n}\left|a_{j i}\right| L_{i}-\frac{p(p-1)}{2} e_{i}-\frac{(p-1)(p-2)}{2} l_{i}\right\}>0, \\
k_{2}= & \max _{1 \leq i \leq n}\left\{N_{i} \sum_{j=1}^{n}\left|b_{j i}\right|+(p-1) l_{i}\right\},
\end{aligned}
$$

(i) there exist $\sigma>0, \lambda>0$ such that $-k_{1}+\frac{k_{2} e^{\lambda \tau}}{d_{k-1}^{p}} \leq \sigma-\lambda$;

(ii) $p \ln d_{k-1}<-(\sigma+\lambda)\left(t_{k}-t_{k-1}\right), k \in \mathbb{N}$, then the equilibrium point of (2.1) is pth moment exponentially stable.

Proof We define a Lyapunov function $V(t, y(t))=\sum_{i=1}^{n}\left|y_{i}(t)\right|^{p}=\|y(t)\|^{p}$. Let $t \geq t_{0}$ and $t \in\left[t_{k-1}, t_{k}\right)$, then we can get the operator $\mathcal{L} V(t, y)$ associated with the system (2.4) of the form as follows:

$$
\begin{aligned}
& \mathcal{L} V(t, y)=p \sum_{i=1}^{n}\left|y_{i}(t)\right|^{p-1} \operatorname{sgn}\left(y_{i}(t)\right)\left[-c_{i} y_{i}(t)+\sum_{j=1}^{n} a_{i j} \tilde{f}_{j}\left(y_{j}(t)\right)+\sum_{j=1}^{n} b_{i j} \tilde{g}_{j}\left(y_{j}\left(t-\tau_{j}(t)\right)\right)\right] \\
& +\frac{p(p-1)}{2} \sum_{i=1}^{n}\left|y_{i}(t)\right|^{p-2} \sum_{l=1}^{m} \tilde{\sigma}_{i l}^{2}\left(t, y_{i}(t), y_{i}\left(t-\tau_{i}(t)\right)\right) \\
& \leq-p \sum_{i=1}^{n} c_{i}\left|y_{i}(t)\right|^{p}+p \sum_{i=1}^{n} \sum_{j=1}^{n}\left|a_{i j}\right| L_{j}\left|y_{i}(t)\right|^{p-1}\left|y_{j}(t)\right| \\
& +p \sum_{i=1}^{n} \sum_{j=1}^{n}\left|b_{i j}\right| N_{j}\left|y_{i}(t)\right|^{p-1}\left|y_{j}\left(t-\tau_{j}(t)\right)\right| \\
& +\frac{p(p-1)}{2} \sum_{i=1}^{n}\left|y_{i}(t)\right|^{p-2}\left(e_{i}\left|y_{i}(t)\right|^{2}+l_{i}\left|y_{i}\left(t-\tau_{i}(t)\right)\right|^{2}\right) \\
& \leq-p \sum_{i=1}^{n} c_{i}\left|y_{i}(t)\right|^{p}+\sum_{i=1}^{n} \sum_{j=1}^{n}\left|a_{i j}\right| L_{j}\left((p-1)\left|y_{i}(t)\right|^{p}+\left|y_{j}(t)\right|^{p}\right) \\
& +\sum_{i=1}^{n} \sum_{j=1}^{n}\left|b_{i j}\right| N_{j}\left((p-1)\left|y_{i}(t)\right|^{p}+\left|y_{j}\left(t-\tau_{j}(t)\right)\right|^{p}\right) \\
& +\frac{p(p-1)}{2} \sum_{i=1}^{n} e_{i}\left|y_{i}(t)\right|^{p}+\frac{(p-1)}{2} \sum_{i=1}^{n} l_{i}\left((p-2)\left|y_{i}(t)\right|^{p}+2\left|y_{i}\left(t-\tau_{i}(t)\right)\right|^{p}\right) \\
& =-\sum_{i=1}^{n}\left[p c_{i}-(p-1) \sum_{j=1}^{n}\left(L_{j}\left|a_{i j}\right|+N_{j}\left|b_{i j}\right|\right)-\sum_{j=1}^{n}\left|a_{j i}\right| L_{i}-\frac{p(p-1)}{2} e_{i}\right]\left|y_{i}(t)\right|^{p} \\
& -\sum_{i=1}^{n} \frac{(p-1)(p-2)}{2} l_{i}\left|y_{i}(t)\right|^{p}+\sum_{i=1}^{n}\left[N_{i} \sum_{j=1}^{n}\left|b_{j i}\right|+(p-1) l_{i}\right]\left|y_{i}\left(t-\tau_{i}(t)\right)\right|^{p} \\
& \leq-k_{1} V(t, y(t))+k_{2} \sup _{t-\tau \leq s \leq t} V(s, y(s)) \text {, }
\end{aligned}
$$


where

$$
\begin{aligned}
k_{1}= & \min _{1 \leq i \leq n}\left\{p c_{i}-(p-1) \sum_{j=1}^{n}\left(L_{j}\left|a_{i j}\right|+N_{j}\left|b_{i j}\right|\right)\right. \\
& \left.-\sum_{j=1}^{n}\left|a_{j i}\right| L_{i}-\frac{p(p-1)}{2} e_{i}-\frac{(p-1)(p-2)}{2} l_{i}\right\}, \\
k_{2}= & \max _{1 \leq i \leq n}\left\{N_{i} \sum_{j=1}^{n}\left|b_{j i}\right|+(p-1) l_{i}\right\} .
\end{aligned}
$$

Let $\gamma=\inf _{k \in \Lambda} \frac{1}{d_{k-1}^{p}}$, there exist $\sigma>0, \lambda>0$ such that

$$
-k_{1}+k_{2} \gamma e^{\lambda \tau} \leq-k_{1}+\frac{k_{2} e^{\lambda \tau}}{d_{k-1}^{p}} \leq \sigma-\lambda
$$

and

$$
\ln \gamma+\lambda \tau-(\sigma+\lambda)\left(t_{k}-t_{k-1}\right)>0
$$

Hence, we can choose $M \geq 1$ such that

$$
e^{(\sigma+\lambda)\left(t_{1}-t_{0}\right)} \leq M \leq \gamma e^{\lambda \tau} .
$$

For convenience, we denote that $\phi(s)=\psi(s)-x^{*}$ for $s \in[-\tau, 0)$.

It is obvious that

$$
\|\phi\|_{\tau}^{p} \leq\|\phi\|_{\tau}^{p} e^{\sigma\left(t_{1}-t_{0}\right)} \leq M\|\phi\|_{\tau}^{p} e^{-\lambda\left(t_{1}-t_{0}\right)} .
$$

Now, we should prove

$$
E\|y(t)\|^{p} \leq M\|\phi\|_{\tau}^{p} e^{-\lambda\left(t-t_{0}\right)}, \quad \forall t \geq t_{0} .
$$

Firstly, we prove when $t \in\left[t_{0}, t_{1}\right)$,

$$
E V(t, y(t))=E\|y(t)\|^{p} \leq M\|\phi\|_{\tau}^{p} e^{-\lambda\left(t_{1}-t_{0}\right)} \leq M\|\phi\|_{\tau}^{p} e^{-\lambda\left(t-t_{0}\right)} .
$$

If (3.7) is not true, there exists $\bar{t} \in\left[t_{0}, t_{1}\right)$ such that

$$
\begin{aligned}
E V(\bar{t}, y(\bar{t})) & >M\|\phi\|_{\tau}^{p} e^{-\lambda\left(t_{1}-t_{0}\right)}>\|\phi\|_{\tau}^{p} e^{\sigma\left(t_{1}-t_{0}\right)} \\
& >\|\phi\|_{\tau}^{p} \geq E V\left(t_{0}+s, y\left(t_{0}+s\right)\right), \quad s \in[-\tau, 0] .
\end{aligned}
$$

Since $V(t, y(t))$ is continuous on $\left[t_{0}, t_{1}\right)$, which implies that there exists $\hat{t} \in\left[t_{0}, \bar{t}\right)$ such that

$$
E V(\hat{t}, y(\hat{t}))=M\|\phi\|_{\tau}^{p} e^{-\lambda\left(t_{1}-t_{0}\right)}
$$


and

$$
E V(t, y(t)) \leq E V(\hat{t}, y(\hat{t})), \quad \forall t \in\left[t_{0}-\tau, \hat{t}\right),
$$

then there exists some $\tilde{t} \in\left[t_{0}, \hat{t}\right)$ satisfying

$$
E V(\tilde{t}, y(\tilde{t}))=\|\phi\|_{\tau}^{p}
$$

and

$$
E V(t, y(t)) \geq E V(\tilde{t}, y(\tilde{t})), \quad \forall t \in(\tilde{t}, \hat{t})
$$

Hence, for any $s \in[-\tau, 0], t \in(\tilde{t}, \hat{t})$,

$$
E V(t+s, y(t+s)) \leq E V(\hat{t}, y(\hat{t}))<\gamma e^{\lambda \tau}\|\phi\|_{\tau}^{p} e^{-\lambda\left(t_{1}-t_{0}\right)}<\gamma e^{\lambda \tau}\|\phi\|_{\tau}^{p} \leq \gamma e^{\lambda \tau} \mathbb{E} V(t, y(t)) .
$$

By (3.1) and (3.2), we get

$$
E \mathcal{L} V(t, y) \leq\left(-k_{1}+k_{2} \gamma e^{\lambda \tau}\right) E V(t, y(t)) \leq(\sigma-\lambda) E V(t, y(t)), \quad \forall t \in(\tilde{t}, \hat{t})
$$

Then

$$
E V(\hat{t}, y(\hat{t})) \leq E V(\tilde{t}, y(\tilde{t})) e^{(\sigma-\lambda)(\hat{t}-\tilde{t})}<\|\phi\|_{\tau}^{p} e^{\sigma\left(t_{1}-t_{0}\right)} \leq M\|\phi\|_{\tau}^{p} e^{-\lambda\left(t_{1}-t_{0}\right)}=E V(\hat{t}, y(\hat{t})),
$$

which is a contradiction. Hence, (3.7) holds.

Next, we will show

$$
E V(t, y(t)) \leq M\|\phi\|_{\tau}^{p} e^{-\lambda\left(t-t_{0}\right)}, \quad t \in\left[t_{k-1}, t_{k}\right), k \in \Lambda
$$

Assuming (3.9) holds for $k=1,2, \ldots, m$, we shall show that it holds for $k=m+1$, i.e.,

$$
E V(t, y(t))=E\|y(t)\|^{p} \leq M\|\phi\|_{\tau}^{p} e^{-\lambda\left(t-t_{0}\right)}, \quad \forall t \in\left[t_{m}, t_{m+1}\right) .
$$

Suppose (3.10) is not true. Then we define $\bar{t} \in\left[t_{m}, t_{m+1}\right)$ such that

$$
E V(\bar{t}, y(\bar{t}))>M\|\phi\|_{\tau}^{p} e^{-\lambda\left(t-t_{0}\right)} .
$$

From (H3) we get

$$
\begin{aligned}
E V\left(t_{m}, y\left(t_{m}\right)\right) & =\sum_{i=1}^{n} E\left|y_{i}\left(t_{m}^{-}\right)+\tilde{p}_{i}\left(y_{i}\left(t_{m}^{-}\right)+x_{i}^{*}\right)\right|^{p}=E \sum_{i=1}^{n}\left|1-\beta_{i m}\right|^{p}\left|y_{i}\left(t_{m}^{-}\right)\right|^{p} \\
& \leq E \sum_{i=1}^{n} d_{m}^{p}\left|y_{i}\left(t_{m}^{-}\right)\right|^{p}=d_{m}^{p} E V\left(t_{m}^{-}, y\left(t_{m}^{-}\right)\right) \leq d_{m}^{p} M\|\phi\|_{\tau}^{p} e^{-\lambda\left(t_{m}-t_{0}\right)} \\
& =d_{m}^{p} M\|\phi\|_{\tau}^{p} e^{\lambda\left(\bar{t}-t_{m}\right)} e^{-\lambda\left(\bar{t}-t_{0}\right)}<d_{m}^{p} M\|\phi\|_{\tau}^{p} e^{\lambda\left(t_{m+1}-t_{m}\right)} e^{-\lambda\left(\bar{t}-t_{0}\right)} \\
& <M\|\phi\|_{\tau}^{p} e^{-\lambda\left(\bar{t}-t_{0}\right)} \leq M\|\phi\|_{\tau}^{p} e^{-\lambda\left(t_{m}-t_{0}\right)},
\end{aligned}
$$


which implies $\bar{t} \in\left(t_{m}, t_{m+1}\right)$. Let

$$
\bar{t}=\inf \left\{t \in\left(t_{m}, t_{m+1}\right): E V(\bar{t}, y(\bar{t}))=M\|\phi\|_{\tau}^{p} e^{-\lambda\left(\bar{t}-t_{0}\right)}\right\},
$$

then for $t \in\left(t_{m}-\tau, \bar{t}\right)$, we can get $E V(t, y(t)) \leq E V(\bar{t}, y(\bar{t}))$.

Hence, there exists $t^{*} \in\left(t_{m}, \bar{t}\right)$ such that

$$
E V\left(t^{*}, y\left(t^{\prime \prime}\right)\right)=d_{m}^{p} M\|\phi\|_{\tau}^{p} e^{-\lambda\left(\bar{t}-t_{0}\right)} e^{\lambda\left(t_{m+1}-t_{m}\right)}
$$

and

$$
E V(t, y(t)) \geq E V\left(t^{*}, y\left(t^{*}\right)\right), \quad t \in\left[t^{*}, \bar{t}\right) .
$$

On the other hand, for any $t \in\left[t^{*}, \bar{t}\right), s \in[-\tau, 0]$, either $t+s \in\left[t_{m}-\tau, t_{m}\right)$ or $t+s \in\left[t_{m}, \bar{t}\right]$.

If $t+s \in\left[t_{m}-\tau, t_{m}\right)$, we can obtain

$$
E V(t+s, y(t+s)) \leq M\|\phi\|_{\tau}^{p} e^{-\lambda\left(t+s-t_{0}\right)}<M\|\phi\|_{\tau}^{p} e^{\lambda \tau} e^{-\lambda\left(\bar{t}-t_{0}\right)} e^{\lambda\left(t_{m+1}-t_{m}\right)}=\frac{e^{\lambda \tau}}{d_{m}^{p}} E V\left(t^{\prime \prime}, y\left(t^{\prime \prime}\right)\right) .
$$

If $t+s \in\left[t_{m}, \bar{t}\right]$, we can get

$$
E V(t+s, y(t+s)) \leq E V(\bar{t}, y(\bar{t}))<\frac{e^{\lambda \tau}}{d_{m}^{p}} E V\left(t^{*}, y\left(t^{*}\right)\right) .
$$

Then for any $s \in[-\tau, 0]$, we get

$$
E V(t+s, y(t+s)) \leq \frac{e^{\lambda \tau}}{d_{m}^{p}} E V\left(t^{*}, y\left(t^{*}\right)\right) \leq \frac{e^{\lambda \tau}}{d_{m}^{p}} E V(t, y(t)), \quad t \in\left[t^{*}, \bar{t}\right) .
$$

Hence,

$$
\mathcal{L} V(t, y) \leq\left(-k_{1}+\frac{k_{2} e^{\lambda \tau}}{d_{m}^{p}}\right) E V\left(t^{*}, y\left(t^{*}\right)\right) \leq(\sigma-\lambda) E V(t, y(t)) .
$$

Then

$$
E V(\bar{t}, y(\bar{t})) \leq E V\left(t^{*}, y\left(t^{*}\right)\right) e^{(\sigma-\lambda)\left(\bar{t}-t^{*}\right)}=d_{m}^{p} M\|\phi\|_{\tau}^{p} e^{-\lambda\left(\bar{t}-t_{0}\right)} e^{\lambda\left(t_{m+1}-t_{m}\right)} e^{(\sigma-\lambda)\left(\bar{t}-t^{*}\right)} .
$$

From the condition (ii), it is obvious that

$$
d_{m}^{p} e^{\lambda\left(t_{m+1}-t_{m}\right)} e^{(\sigma-\lambda)\left(\bar{t}-t^{*}\right)}<1
$$

Hence,

$$
E V(\bar{t}, y(\bar{t}))<M\|\phi\|_{\tau}^{p} e^{-\lambda\left(\bar{t}-t_{0}\right)}=E V(\bar{t}, y(\bar{t})),
$$

which is a contradiction. Hence, (3.10) holds. 
By induction, we can obtain that (3.9) holds for any $k \in \Lambda$, i.e.,

$$
E V(t, y(t)) \leq M\|\phi\|_{\tau}^{p} e^{-\lambda\left(t-t_{0}\right)}, \quad \forall t \geq t_{0},
$$

which implies that the equilibrium point of the impulsive system (2.1) is $p$ th moment exponentially stable. This completes the proof of the theorem.

Theorem 3.2 Assume that (H1)-(H3) hold, $\mu_{i}(i \in \Lambda)>0$,

(i) if there exist $\sigma>0, \lambda>0$ such that $-k_{1}+\frac{k_{2}}{e}{ }^{\lambda \tau} d_{k-1}^{p} \leq \sigma-\lambda$;

(ii) $p \ln d_{k-1}<-(\sigma+\lambda)\left(t_{k}-t_{k-1}\right), k \in \mathbb{N}$,

where

$$
\begin{aligned}
k_{1}= & \min _{1 \leq i \leq n}\left\{p c_{i}-(p-1) \sum_{j=1}^{n}\left(L_{j}\left|a_{i j}\right|+N_{j}\left|b_{i j}\right|\right)\right. \\
& \left.-\sum_{j=1}^{n} \frac{\mu_{j}}{\mu_{i}}\left|a_{j i}\right| L_{i}-\frac{p(p-1)}{2} e_{i}-\frac{(p-1)(p-2)}{2} l_{i}\right\}>0, \\
k_{2}= & \max _{1 \leq i \leq n}\left\{N_{i} \sum_{j=1}^{n} \frac{\mu_{j}}{\mu_{i}}\left|b_{j i}\right|+(p-1) l_{i}\right\},
\end{aligned}
$$

then the equilibrium point of the system (2.1) is pth moment exponentially stable.

Proof Let $V(t, y(t))=\sum_{i=1}^{n} \mu_{i}\left|y_{i}(t)\right|^{p}$, the proof of the theorem is similar to that of Theorem 3.1 hence it is omitted.

Corollary 3.3 Assume that (H1)-(H3) hold, $\mu_{i}(i \in \Lambda)>0$,

$$
\begin{aligned}
& k_{1}=\min _{1 \leq i \leq n}\left\{2 c_{i}-\sum_{j=1}^{n}\left(L_{j}\left|a_{i j}\right|+N_{j}\left|b_{i j}\right|\right)-\sum_{j=1}^{n} \frac{\mu_{j}}{\mu_{i}}\left|a_{j i}\right| L_{i}-e_{i}\right\}>0, \\
& k_{2}=\max _{1 \leq i \leq n}\left\{N_{i} \sum_{j=1}^{n} \frac{\mu_{j}}{\mu_{i}}\left|b_{j i}\right|+l_{i}\right\},
\end{aligned}
$$

(i) if there exist $\sigma>0, \lambda>0$ such that $-k_{1}+\frac{k_{2} e^{\lambda \tau}}{d_{k-1}^{2}} \leq \sigma-\lambda$;

(ii) $2 \ln d_{k-1}<-(\sigma+\lambda)\left(t_{k}-t_{k-1}\right), k \in \mathbb{N}$,

then the equilibrium point of the system (2.1) is exponentially stable in mean square.

Remark 3.4 In many stability results for stochastic cellular neural networks, $E \mathcal{L} V \leq 0$ is an important condition for their conclusions [13-15], which means that the origin systems without impulses need to be stable. However, by constructing the impulses, we do not need this condition to ensure the equilibrium point of the impulsive system (2.1) is $p$ th moment exponentially stable. Our results show that impulses play an important role in the $p$ th moment exponential stability for the stochastic cellular neural network with time delay, even if the corresponding systems may be unstable themselves. It should be mentioned that our results develop an effective impulse control strategy to stabilize underlying retarded cellular neural networks. And it is particularly meaningful for some practical applications. 
Remark 3.5 It is important to emphasize that, in contrast to some existing exponential stability results, see $[6,11,12,19]$, the condition $k_{1}>k_{2}$ is needed to ensure the equilibrium point of the system (2.1) is $p$ th moment exponentially stable, while in our paper we omit it and obtain the results.

\section{Illustrative example}

In the following, we will give an example to illustrate the advantages of our results.

Example 1 Consider the following model:

$$
\left\{\begin{aligned}
d x_{i}(t)= & {\left[-c_{i} x_{i}(t)+\sum_{j=1}^{2} a_{i j} f_{j}\left(x_{j}(t)\right)+\sum_{j=1}^{2} b_{i j} g_{j}\left(x_{j}\left(t-\tau_{j}(t)\right)\right)\right] d t } \\
& +\sigma_{i}\left(t, x_{i}(t), x_{i}\left(t-\tau_{i}(t)\right)\right) d w(t), \quad t \neq t_{k}, \\
x_{i}\left(t_{k}\right)= & \left(1-\beta_{i k}\right) x_{i}\left(t_{k}^{-}\right) k \in \mathbb{Z}^{+}, \quad i \in \Lambda=(1,2)
\end{aligned}\right.
$$

where $f_{i}(x)=g_{i}(x)=\tanh (x), 0 \leq \tau_{i}(t) \leq \tau=0.5, t_{k}-t_{k-1}=0.1, x_{1}\left(t_{k}\right)=\frac{x_{1}\left(t_{k}^{-}\right)}{3}, x_{2}\left(t_{k}\right)=\frac{x_{2}\left(t_{k}^{-}\right)}{4}$. $C_{2 \times 1}=\left(\begin{array}{l}2 \\ 2\end{array}\right),\left(a_{i j}\right)_{2 \times 2}=\left(\begin{array}{cc}0.5 & -0.8 \\ 0.3 & 0.6\end{array}\right),\left(b_{i j}\right)_{2 \times 2}=\left(\begin{array}{cc}0.2 & -0.3 \\ -0.1 & 0.4\end{array}\right)$.

Obviously, $L_{i}=N_{i}=1, e_{i}=0, l_{i}=1(i=1,2), \beta_{1 k}=2 / 3, \beta_{2 k}=3 / 4$.

Let $d_{k}=2 / 3, \sigma=3.4, \lambda=0.2, \mu_{1}=\mu_{2}$. Then for $p=2$, we can get $k_{1}=\min (1.4,1.2)=1.2>$ $0, k_{2}=\max (1.3,1.7)=1.7,-k_{1}+k_{2} \frac{e^{\lambda \tau}}{d_{k}^{p}}=-1.2+1.7 \times \frac{e^{0.2 \times 0.5}}{(2 / 3)^{2}}=3.027<\sigma-\lambda=3.4-0.2=3.2$, $2 \ln d_{k-1}=2 \ln (2 / 3)=-0.811<-(\sigma+\lambda)\left(t_{k}-t_{k_{1}}\right)=-3.6 \times 0.1=-0.36$.

All conditions of Corollary 3.3 are satisfied, then the equilibrium point is exponentially stable in mean square.

Remark 4.1 If $\mu_{1}=\mu_{2}$, we have computed $k_{1}=1.2, k_{2}=1.7$. If $\mu_{1} \neq \mu_{2}$, set $\frac{\mu_{2}}{\mu_{1}}=\alpha$, then $k_{1}=\min \{1.7-0.3 \alpha, 2-0.8 / \alpha\}, k_{2}=\max \{1.2+0.1 \alpha, 1.4+0.3 / \alpha\}$. If $\mu_{1}<\mu_{2}$, then $\alpha>1$, we can compute $k_{1}<1.4$ and $k_{2}>1.4$; if $\mu_{1}>\mu_{2}$, then $0<\alpha<1, k_{1}<1.2$ and $k_{2}>1.4$. Hence, in either case, we always have $k_{1}<k_{2}$, so the exponential stability in mean square of the system (4.1) cannot be derived by applying the corresponding exponential stability result for cellular neural networks given in the literature $[6,11,12,19]$, since $k_{1}>k_{2}$ is not satisfied.

Remark 4.2 Since $\rho\left[C^{-1}\left(M M_{1} K+M M_{2} K+N N_{1}+N N_{2}\right)\right]=33.635$, where $\rho\left[C^{-1}\left(M M_{1} K+\right.\right.$ $\left.\left.M M_{2} K+N N_{1}+N N_{2}\right)\right]$ was defined in [8], the condition $\rho\left[C^{-1}\left(M M_{1} K+M M_{2} K+N N_{1}+\right.\right.$ $\left.\left.N N_{2}\right)\right] \leq 1$ is not satisfied. Hence, the results in [8] are useless to judge the exponential stability of the system (4.1).

Competing interests

The authors declare that they have no competing interests.

Authors' contributions

$\mathrm{XL}$ completed the proof and wrote the initial draft. JZ gave some suggestions on the amendment. XL then finalized the manuscript. Correspondence was mainly done by EZ. All authors read and approved the final manuscript.

\section{Acknowledgements}

The authors would like to thank the editor and the anonymous referees for their detailed comments and valuable suggestions which considerably improved the presentation of this paper. This work was supported in part by the National Natural Science Foundation of China under Grant No. 11101054, the Hunan Provincial Natural Science Foundation of China under Grant No. 12jj4005, the Open Fund Project of Key Research Institute of Philosophies and Social Sciences in Hunan Universities under Grant No. 11FEFM11 and the Scientific Research Funds of Hunan Provincial Science and Technology Department of China under Grant No. 2012SK3096. 


\section{References}

1. Chua, LO, Yang, L: Cellular neural networks: theory. IEEE Trans. Circuits Syst. 35, 1257-1272 (1988)

2. Chua, LO, Yang, L: Cellular neural networks: applications. IEEE Trans. Circuits Syst. 35, 1273-1290 (1988)

3. $\mathrm{Wu}, \mathrm{B}, \mathrm{Liu}, \mathrm{Y}, \mathrm{Lu}, \mathrm{J}$ : New results on global exponential stability for impulsive cellular neural networks with any bounded time-varying delays. Math. Comput. Model. 55, 837-843 (2012)

4. Ahmada, S, Stamova, IM: Global exponential stability for impulsive cellular neural networks with time-varying delays. Nonlinear Anal. 69, 786-795 (2008)

5. Zhao, H, Cao, J: New conditions for global exponential stability of cellular neural networks with delays. Neural Netw. $18,1332-1340(2005)$

6. Huang, C, He, Y, Huang, L, Zhu, W: pth moment stability analysis of stochastic recurrent neural networks with time-varying delays. Inf. Sci. 178, 2194-2203 (2008)

7. Huang, C, Cao, J: Almost sure exponential stability of stochastic cellular neural networks with unbounded distributed delays. Neurocomputing 72, 3352-3356 (2009)

8. Sun, Y, Cao, J: pth moment exponential stability of stochastic recurrent neural networks with time-varying delays. Nonlinear Anal., Real World Appl. 8, 1171-1185 (2007)

9. Wu, S, Li, C, Liao, X, Duan, S: Exponential stability of impulsive discrete systems with time delay and applications in stochastic neural networks: a Razumikhin approach. Neurocomputing 82, 29-36 (2012)

10. Chen, L, Wu, R, Pan, D: Mean square exponential stability of impulsive stochastic fuzzy cellular neural networks with distributed delays. Expert Syst. Appl. 38, 6294-6299 (2011)

11. Li, X: Existence and global exponential stability of periodic solution for delayed neural networks with impulsive and stochastic effects. Neurocomputing 73, 749-758 (2010)

12. $\mathrm{Li}, \mathrm{X}, \mathrm{Fu}, \mathrm{X}$ : Synchronization of chaotic delayed neural networks with impulsive and stochastic perturbations. Commun. Nonlinear Sci. Numer. Simul. 16, 885-894 (2011)

13. Mao, X: Exponential Stability of Stochastic Differential Equations. Marcel Dekker, New York (1994)

14. Li, X, Fu, X: Stability analysis of stochastic functional differential equations with infinite delay and its application to recurrent neural networks. J. Comput. Appl. Math. 234, 407-417 (2010)

15. Yang, Z, Zhu, E, Xu, Y, Tan, Y: Razumikhin-type theorems on exponential stability of stochastic functional differential equations with infinite delay. Acta Appl. Math. 111, 219-231 (2010)

16. Peng, S, Zhang, Y: Razumikhin-type theorems on $p$ th moment exponential stability of impulsive stochastic delay differential equations. IEEE Trans. Autom. Control 55, 1917-1922 (2010)

17. Li, X, Zou, J, Zhu, E: Pth moment exponential stability of impulsive stochastic neural networks with mixed delays. Math. Probl. Eng. 2012, Article ID 175934 (2012). doi:10.1155/2012/175934

18. Mao, X: Stochastic Differential Equations and Applications. Horwood Publication, Chichester (1997)

19. Wan, L, Zhou, Q: Attractor and ultimate boundedness for stochastic cellular neural networks with delays. Nonlinear Anal., Real World Appl. 12, 2561-2566 (2011)

doi:10.1186/1687-1847-2013-6

Cite this article as: Li et al.: The $p$ th moment exponential stability of stochastic cellular neural networks with

impulses. Advances in Difference Equations 2013 2013:6.

\section{Submit your manuscript to a SpringerOpen ${ }^{\circ}$ journal and benefit from:}

- Convenient online submission

- Rigorous peer review

- Immediate publication on acceptance

Open access: articles freely available online

- High visibility within the field

- Retaining the copyright to your article 